\title{
Frontières
}

\section{Le menaçant et la construction de l'innocence des victimes}

Guerre, génocide et terrorisme

\section{Klaus Dammann}

Volume 15, numéro 2, printemps 2003

Guerre, mort amère

URI : https://id.erudit.org/iderudit/1073812ar

DOI : https://doi.org/10.7202/1073812ar

Aller au sommaire du numéro

Éditeur(s)

Université du Québec à Montréal

ISSN

1180-3479 (imprimé)

1916-0976 (numérique)

Découvrir la revue

Citer cet article

Dammann, K. (2003). Le menaçant et la construction de l'innocence des victimes : guerre, génocide et terrorisme. Frontières, 15(2), 6-11.

https://doi.org/10.7202/1073812ar
Résumé de l'article

Cette analyse utilise des distinctions empruntées aux théories développées par Niklas Luhmann, un des sociologues contemporains les plus brillants, en particulier la distinction entre sémantique et structure sociale, permettant de comprendre comment la guerre, le génocide et le terrorisme sont construits par l'intermédiaire, d'une part, d'un code qui englobe la menace et, d'autre part, d'une sémantique d'innocence des victimes. 


\section{Résumé}

Cette analyse utilise des distinctions empruntées aux théories développées par Niklas Luhmann, un des sociologues contemporains les plus brillants, en particulier la distinction entre sémantique et structure sociale, permettant de comprendre comment la guerre, le génocide et le terrorisme sont construits par l'intermédiaire, d'une part, d'un code qui englobe la menace et, d'autre part, d'une sémantique $d$ 'innocence des victimes.

Mots clés : terrorisme - menace guerre - militaire - génocide - victimes communication - sémantique différenciation fonctionnelle.

\section{Abstract \\ This analysis uses distinctions (especially: semantics/social structure) of the theory of one of the most brilliant contem- porary sociologists, Niklas Luhmann, towards an understanding of how war- fare, genocide and terrorism are con- structed by means of a code that contains threat and of a semantics of innocence of victims.}

Key words : terrorism - threat warfare-military-genocide-victims communication - semantics - functional differentiation.

\section{Le menaçant et la construction de l'innocence des victimes GUERRE, GÉNOCIDE ET TERRORISME}

\author{
Klaus Dammann, Ph.D., \\ professeur, Faculté de sociologie, \\ Université de Bielefeld, Allemagne.
}

Il y a apparition de la peur lors de périodes de durée variable que l'on décrit après coup comme agitées par des guerres, des génocides ou des actes terroristes. C'est ainsi que, depuis plus de deux siècles, la terreur et la victimisation sont associées dans un climat social de meurtres en série commis par des organisations structurées sur le mode militaire ou policier, sur le mode de la guérilla, sur le mode maffieux ou même judiciaire (voir Sassòli et Hébert, dans ce numéro). Il n'y a donc pas que le terrorisme qui suscite la terreur, la peur, l'anxiété.

La sociologie aborde ces questions parfois avec des outils un peu démodés (théories de Marx, Durkheim, Weber, Mead, Parsons). Ce sont les approches dites néoclassiques qui sont évidemment les plus intéressantes pour un public composé de non-spécialistes mais convaincu de la possibilité d'un progrès dans toutes les sciences, sciences sociales incluses. Parmi les approches sociologiques contemporaines, qui visent à couvrir tous les phénomènes sociaux, la sociologie néo-wébérienne de Pierre Bourdieu (1930-2002) est certainement plus connue dans le monde francophone que celle de Niklas Luhmann (1927-1998) $)^{1}$, qui pourrait être facilement considéré comme un antipode de Bourdieu. Je vais essayer de montrer comment, à la lumière des distinctions qui caractérisent son œuvre, ce ne sont pas seulement la menace et la peur qui sont utilisées pour déchiffrer le terrorisme. La sémantique mondiale d'aujourd'hui a recours à des distinctions directrices différentes. On peut dire la même chose de la structure sociale.

\section{LE MENAÇANT ET LA PEUR}

La peur ne peut être ramenée à un état psychique que pour ceux qui l'éprouvent et pour la psychologie dans sa dimension scientifique. Les autres disciplines, ainsi que la communication des non-spécialistes, ne disposent pas des outils méthodologiques nécessaires pour faire la distinction entre le psychique et le social. Quand par exemple les historiens parlent de "mentalité », ou quand un mari qualifie l'état psychique de son épouse en lui disant: "tu as peur", leurs observations reposent sur la communication. Des mots comme «mentalité ", «dans la tête", "psychiquement », etc., ne sont que des mots, qui envoient parfois les individus chez leur psychologue ou même leur psychiatre, mais qui ne doivent pas être confondus avec le concept de "psyche» utilisé en psychologie.

Dans la communication (qui constitue, selon Luhmann, «le social», objet de la sociologie, alors que les états psychiques 
constituent l'objet de la psychologie), on distingue les dangers des risques. Être tué par un séisme, une inondation, une action génocidale ou terroriste est le plus souvent un danger dans la mesure où la menace de mort est attribuée à la décision d'autres personnes ou d'autres choses - à des acteurs (hommes, organisations, dieux) ou à la nature. On a affaire à une situation tout à fait différente quand la vie est menacée par la consommation de tabac, par une trop grande vitesse sur la route ou par la nonintervention gouvernementale et quand le raccourcissement de la vie est attribué à la décision «volontaire » de celui qui fume, qui roule trop vite ou du gouvernement qui refuse d'intervenir. Le fumeur et le conducteur risquent leur propre vie et le gouvernement, la vie des citoyens. On peut donc dire que ce qu'on a qualifié de société $\mathrm{du}$ risque est le résultat d'une évolution vers une société dans laquelle de plus en plus d'événements peuvent être attribués à des décisions. Cet état de chose est une conséquence du progrès technique. Avant l'invention du parapluie, il était dangereux de quitter la maison quand il pleuvait, tandis qu'après cette invention, on risque de se mouiller - et l'on risque de perdre le parapluie (Luhmann, 1993). Dans la communication, on constate une tendance à

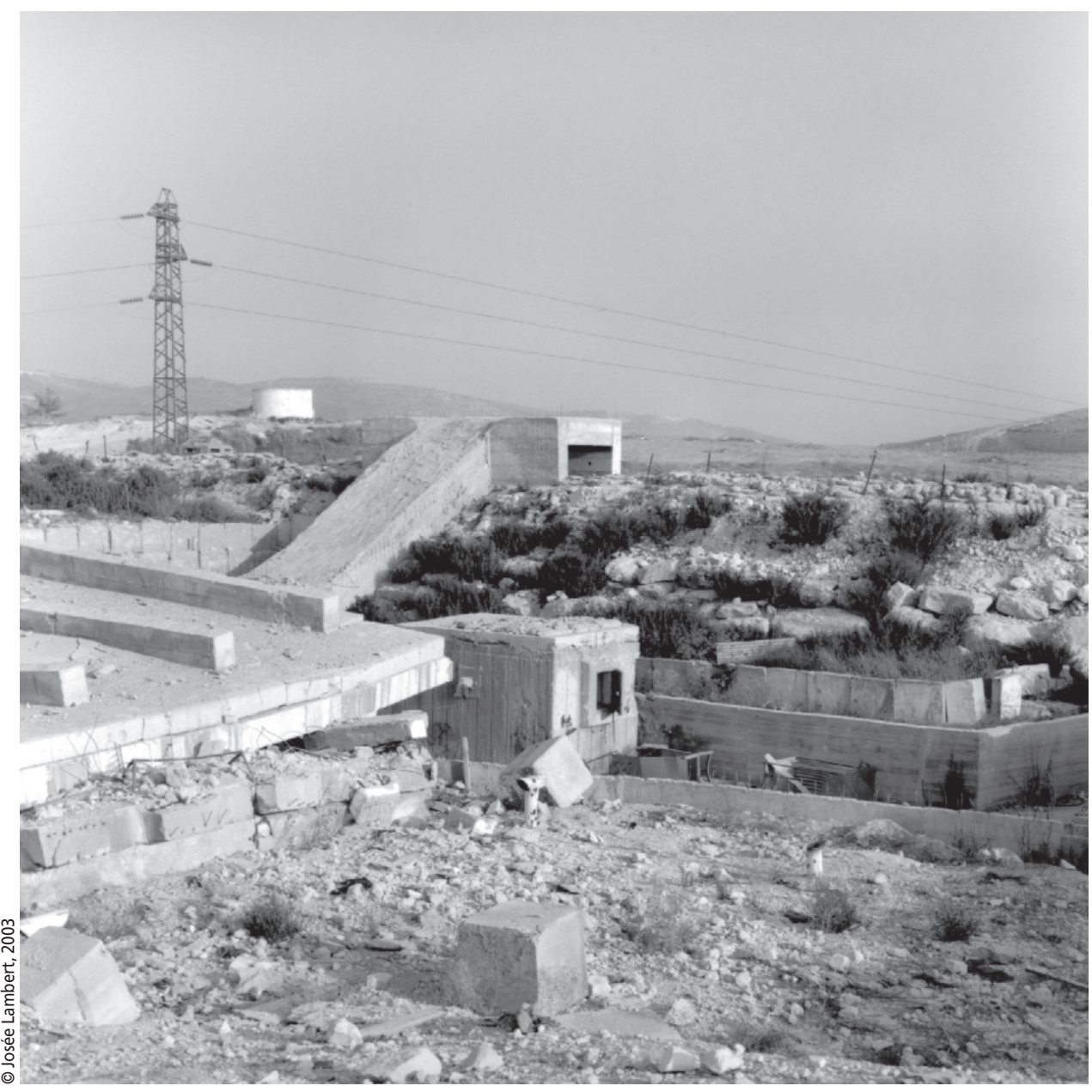

construire un sentiment de peur quand un état évalué d'une façon négative (la mort précoce) n'est pas la conséquence d'une activité volontaire de ceux qui participent à la communication. Ainsi, dans la communication, la perception du danger que comportent les actions militaires, génocidales ou terroristes résulte du fait que ceux qui participent à la communication disposent de trop peu de moyens de les éviter, et surtout d'éviter la mort précoce. C'est pour cela que le terrorisme dont les cibles se limitent à des bâtiments américains et des complexes touristiques, cibles dites soft, en pays de majorité musulmane, dans des lieux où l'on n'a ni les moyens ni l'intention de se rendre, n'est pas perçu comme un danger dans les pays occidentaux, mais plutôt pour la population sur place. Et ce terrorisme ne provoque qu'une communication d'anxiété très limitée parmi ceux qui courent ce risque, c'est-à-dire parmi les organisation terroristes et peut-être leurs membres. Leur risque est différent d'un danger. Bien sûr, une fois la décision prise, il y aura peutêtre du regret s'il y a destruction de l'organisation terroriste ou si ses membres sont tués ou blessés. Ce que l'on qualifie de post-decision regret est un bon indicateur $\mathrm{du}$ risque en cause. 
militaires qui interviennent régulièrement et avec succès pour tout au moins canaliser les pogroms, les riots. L'absence d'organisation est aussi considérée moins dangereuse parce qu'on s'imagine difficilement qu'une foule ou une bande puisse se procurer des armes de technologie avancée ayant une force de destruction étendue. Troisième cause : ces collectifs non organisés n'ayant pas la même capacité qu'une organisation de former des chaînes de décisions, ils ne peuvent pas non plus terroriser les masses en menaçant d'utiliser une technologie moins avancée. Il y a des séries d'attaques réalisées par des moyens relevant d'une technologie peu avancée qui causent de grands nombres de victimes, mais elles sont organisées, au moins dans la mesure où la police et l'armée s'abstiennent d'intervenir. On peut considérer comme des exemples de ce phénomène de meurtre organisé avec des moyens relevant d'une technologie peu avancée la grande famine en Ukraine (génocide attribué au régime stalinien), le pogrom de Lvov, en Ukraine, en 1941 (massacre génocidal auquel des bandes armées se sont laissées inciter par l'armée allemande) et aussi la mort de millions d'autres Juifs qui, durant la Shoah, n'ont pas été victimes des chambres à gaz mais d'attaques d'unités de meurtre armées d'armes de technologie peu avancée. Et enfin, ce qui rend l'organisation meurtrière si efficace - et par là si menaçante -, c'est la mobilité. Tandis que l'action des foules et des bandes qui assassinent reste locale et s'épuise vite, l'organisation permet de semer la terreur sur un territoire plus vaste. Si l'on en croit certains témoins de la terreur au Rwanda en 1994, il y a eu des événements spontanés au niveau local, comme déjà en 1993. Mais il semble que des émetteurs de radio racistes aient incité les Hutus au meurtre et que des unités mobiles paramilitaires aient empêché les Tutsis victimisés de s'enfuir. Ces forces organisées ont donné aux attaques une effroyable efficacité alors que, sans cela, elles seraient restées locales et auraient fait moins de victimes.

\section{QUI DONC EST MENAÇÉ ? LE MILITAIRE ET LES AUTRES CHAMPS SOCIAUX}

L'un des théorèmes les moins contestés en sociologie reconnaît comme un caractère distinctif - exclusif ou supplémentaire de la modernité la formation de sphères sociales fonctionnelles comme l'éducation, le droit, l'économie, la science, les arts, etc., conçues comme des "sphères de valeurs" chez Max Weber, des "champs sociaux» dans l'œuvre de Pierre Bourdieu et des "systèmes fonctionnels" dans celle de Niklas Luhmann.
La communication militaire peut être considérée comme une sphère séparée de la communication politique, économique, légale, etc. La cible sur laquelle porte la menace est tout à fait différente selon qu'il s'agit d'une action guerrière ou d'une action violente non guerrière, par exemple une purification ethnique, qui peut relever $\mathrm{du}$ champ social politique et / ou économique, et / ou religieux. génocide comme meurtre sans but en dehors du meurtre (voir par exemple Bouchereau, 1999).

La terreur entre dans la communication militaire normale dans la mesure où les actions guerrières sont «totalisées». Presque toute action militaire entraîne le risque de ruiner une partie de la population qui n'est pas organisée dans des unités militaires et des organisations infrastructu-

\section{LOIN DE «CHOISIR » DES VICTIMES HUMAINES, LA STRATÉGIE}

TERRORISTE CHOISIT D'IMPRESSIONNER LA POPULATION.

La communication militaire se distingue de toute autre par sa référence au code binaire «détruire ou ne pas détruire la capacité destructive d'une organisation par une contre-organisation ». Au sens étroit du terme menace, ceux qui sont menacés par la force sont donc les organisations adversaires, pas nécessairement les organisations étatiques mais aussi les organisations privées ou parapubliques, comme peuvent l'être une guérilla ou la mafia, etc. al-Qaïda est considéré comme une organisation militaire (plus exactement comme un réseau d'organisations) dans presque toute la communication américaine et alliée. Sa cible n'est pas la population américaine (ou celle de tout l'Occident), mais les organisations politiques du monde américain (ou occidental) qui à la longue doivent être forcées d'abandonner une politique défendant un mode de vie méprisable à ses yeux.

Les épurations ethniques du siècle dernier, qui ont commencé en Afrique du SudOuest (unités militaires allemandes contre Hereros) et dans l'Empire ottoman (unités policières, civiles et militaires contre les chrétiens arméniens) pour culminer dans la Shoah (dont the unique uniqueness consiste dans le caractère organisé des persécutions antisémites), se distinguaient nettement des actions militaires dans lesquelles elles étaient intégrées par la référence à d'autres codes binaires, celui de la politique (être ou ne pas être au pouvoir) et de l'économie (avoir ou non de l'argent). On peut observer ce code dans la cible de la menace: ou bien ce sont des populations qui sont la cible ( émigrez ou vous serez exterminés »), ou bien il n'y a pas de cible de menace du tout: l'émigration est empêchée et toute la population restée sur place est assassinée sans pouvoir faire quoi que ce soit pour éviter ce sort (abandon de ses biens, émigration, etc.). C'est ce qu'on appelle une communication génocidale dans un sens non juridique; c'est le relles comme les voies ferrées, les usines d'armement et l'administration militaire (les cibles de menace). C'est ce qu'on appelle les «dommages collatéraux». Mais il y a surtout deux sortes d'actions qui «totalisent » les victimes en dehors des organisations ciblées en prenant la population dite civile en otage : les bombardements à large périmètre (symbolisés par Belgrade 1941, Dresde 1945, Hiroshima 1945) et la prise d'otages («Nous exécuterons 20 hommes détenus pour chaque personne tuée par la guérilla »), qui était encore largement considérée comme légale pendant la guerre 1939-1945 dans la communication alliée et nazie. La communication, différenciée elle-même en sphères (politique, militaire, économique, religieuse, etc.), considère ces actions comme une communication militaire quand elles sont attribuées à des organisations.

Naturellement la communication légale (une autre sphère!) peut observer cette communication d'une façon différente et d'une manière pluraliste. Dans les années 1970, les avocats des terroristes gauchistes allemands de la RAF ont insisté sur le statut militaire de cette organisation, mais aujourd'hui il y a des juristes qui dans le but d'aider les prisonniers détenus par les Américains comme prisonniers militaires en raison de leur soutien aux luttes d'al-Qaïda cherchent plutôt à présenter leurs actions comme une sorte d'actes criminels sans caractère militaire. Il y a naturellement une troisième solution lorsqu'il s'agit de communication juridique: considérer ces actes comme militaires et illégaux, c'est-à-dire comme des crimes de guerre.

L'observation sociologique (une « observation de second degré ») de l'observation juridique souvent contestée («pluraliste») est encore autre chose que l'observation dans la perspective sociologique de cette communication militaire (qui est peut-être aussi une communication politique, écono- 
mique, religieuse) qui se réalise quand une organisation est en train de tuer et quand une autre organisation cherche à y mettre fin par d'autres actes meurtriers. La communication de premier degré dans une société divisée elle-même en sphères, c'est ce que Luhmann conçoit comme une "structure sociale ». La communication des unités militaires et SS allemandes qui participaient à la Shoah savait quand il s'agissait d'actes militaires - légaux ou non - par exemple quand ces actes s'attaquaient à des individus masculins d'un âge moyen soupçonnés d'être des partisans. Cette communication savait également ce qu'il en était lorsqu'elle devenait politique et économique (par exemple quand des femmes, des enfants et des personnes trop âgées pour être dangereuses étaient exterminés, voir Dammann, 2003). Fin 1941, dans la période qui est jugée par l'historiographie comme ayant abouti à la Shoah, les unités meurtrières faisaient état de cette différence sans aucun scrupule pas seulement dans leurs actes meurtriers, mais même dans les rapports qu'elles rédigeaient. Elles le savaient quand leurs actes franchissaient la frontière entre guerre et génocide. On peut dire la même chose pour les bombardements d'Hiroshima et de Nagasaki, pour les luttes israélo-palestiniennes, pour l'attaque au World Trade Center et pour la lutte qui en a suivi sous le vocable de

\section{LA COMMUNICATION NE PEUT PLUS S'EMPÊCHER}

DE PRENDRE EN COMPTE LA PARTICULARITÉ DES VIOLENCES GUERRIÈRES, GÉNOCIDAIRES ET TERRORISTES.

war on terrorism. La communication non sémantique sait de quoi il s'agit. Si l'on se réfère au code militaire (c'est-à-dire au code binaire «détruire ou ne pas détruire la capacité destructive d'une organisation par une contre-organisation »), il est évident, selon le processus de décision en cause dans les exemples cités, qu'il s'agissait d'actes militaires (peut-être aussi politiques et, dans le cas du World Trade Center, religieux), mais non pas d'actes génocidaires.

\section{LA SÉMANTIQUE, IL S'AGIT D'AUTRE CHOSE :}

LA CONSTRUCTION DE L'INNOCENCE

Quand on considère (toujours d'un point de vue sociologique) non pas cette communication de premier degré (Hiroshima, etc., comme communication militaire) mais la communication de second degré (juridique, littéraire, éducative, voire sociolo- gique), on se place dans le domaine de la sémantique. La distinction entre sémantique et structure sociale prend dans la sociologie de Luhmann la place de distinctions plus anciennes comme celles entre superstructure et base, entre culture et autres structures sociales, entre idées et réalité réalisée, etc. (voir Staeheli, 1997; Stichweh, 2000). La structure sociale peut être identifiée à un niveau opérationnel, celui où se produit la communication ( «where communication just happens ») et la sémantique constitue un niveau différent, celui où il y a observation et autodescription de cette communication ("an observational level, where communication describes itself », Luhmann, 2000, p. 195 ; cf. Luhmann, 1996b, p. 60-69).

La sémantique qui est utilisée pour observer les tueries organisées ne s'intéresse pas à la manière dont les processus de décision définissent eux-mêmes les actes de destruction. Surtout dans le milieu de gauche (dit libéral) des universités américaines, où il y a des voix plus anti-américaines qu'au Canada et en Allemagne, les cas d'Hiroshima et de Dresde sont qualifiés de génocide ( $c f$. Lifton et Markusen, 1990). Et la communication politique et médiatique refuse très souvent de parler de guerres de libération quand des organisations basques, d'Irlande $\mathrm{du}$ Nord ou palestiniennes attaquent des cibles militaires et civiles dans le territoire

d'autres normes, etc.), on les juge plus ou moins coupables (il ne s'agit pas ici de culpabilité dans un sens moral ou juridique!). En revanche, lorsque c'est un caractère ascriptif qui fait d'elles des victimes, elles sont considérées comme tout à fait innocentes. Les victimes des séries de meurtres si visibles au $\mathrm{XX}^{\mathrm{e}}$ siècle comme les catastrophes arménienne (à partir de 1915), juive, tsigane ou encore l'extermination des personnes souffrant de maladies mentales (à partir de 1939) et des Tutsis (1994), sont dans ce sens des victimes innocentes. Elles ont été des victimes parce qu'elles «étaient » des Arméniens, des Juifs, etc., et non pas parce qu'elles ont agi (ou refusé d'agir) d'une manière quelconque.

C'est au moins la position de l'historiographie dominante et - naturellement celle des organisations de victimes. Mais la sémantique d'innocence, qui constitue un des piliers de la sémantique de guerre et de génocide, est suffisamment souple pour permettre à la communication de construire l'histoire des tueries d'une façon différente. Le discours négationniste sur l'arménocide et (moins clair) sur le judéocide le montre bien. En ce qui concerne les bombardements des cibles civiles, on discute pour savoir si ces victimes ont été choisies simplement "parce qu'elles étaient là où elles étaient », c'est-à-dire selon un critère ascriptif. Le contre-argument traite ces victimes civiles comme une extension des organisations contre lesquelles la guerre est menée, ainsi elles ne sont pas «innocentes », même si elles se sont opposées au régime et à la guerre (voir pour la discussion Fein, 1993). Dans le cas de la catastrophe arménienne dans l'Empire ottoman, les autorités turques ainsi que les chercheurs pro-Turcs ont essayé d'en faire une guerre : la déportation des femmes, enfants et personnes âgées, malades et aveugles dans le désert était selon eux une mesure de guerre à la suite d'une insurrection arménienne liant la nation arménienne à l'armée russe qui s'était alors avancée jusqu'au Caucase ${ }^{2}$.

façon moralisante quand la terreur règn Israël, en Palestine, au Kosovo, en Serbie, en Espagne ou bien dans un endroit devenu une cible anti-américaine ou une cible de la lutte «anti-terroriste » américaine.

Cette sémantique par laquelle les concepts de guerre, génocide, terrorisme, peine de mort et autres typologies d'assassinats organisés sont construits se sert de deux distinctions directrices. D'une part, en ce qui concerne les auteurs des actes, la sématique est construite selon la distinction entre organisation et non-organisation (foule, bande, etc.). D'autre part, on crée différentes catégories de victimes en introduisant des critères de sélection. Quand les victimes sont considérées comme présentant des critères d'action (attaque, résistance à

\section{LA SÉMANTIQUE DU TERRORISME : LES VICTIMES SONT-ELLES CHOISIES ?}

Quand on observe le terrorisme en tant que structure sociale, on constate qu'il un cas spécial de la communication militaire et on ne comprend pas très bien comment cette communication peut se distinguer des guerres dites normales ou de toute autre communication dans cette sphère. Dans l'historiographie et la sociologie du terrorisme, on a toujours insisté sur ce point (voir par exemple Wheeler, 1991 ; Silke, 1996). La solution la plus facile est appartient aussi au champ militaire. C'est 
de dire : le terrorisme est la communication militaire qui est décrite comme terroriste par l'observation politique et / ou juridique.

Dans ce cas, c'est la sémantique, cette partie de la communication distincte de la structure sociale, qu'il faut analyser. C'est la sémantique politique et / ou juridique qui construit le terrorisme comme phénomène social. Comment y réussit-elle? Ce n'est pas la distinction entre les critères de choix des victimes (ascriptif ou action) qui est en jeu. Les victimes humaines du terrorisme ne sont des victimes que par raccroc. Le terrorisme ne se définit pas par son besoin de choisir ses victimes. Le terrorisme ne vise ses adversaires (qui sont régulièrement des organisations étatiques) que par le moyen de la propagande, ce qui comprend souvent l'intimidation de la population. C'est la publicité pour la cause du mouvement terroriste qui est importante et non pas les êtres humains qui sont blessés et tués : propaganda by the deed. "Il n'y a pas d'innocents »- c'est comme cela qu'Émile Henry, anarchiste français, se défendait au tribunal après avoir jeté une bombe dans un café parisien (Waldmann, 1998, p. 161). Et comme le disait un spécialiste: "In fact terrorists generally want a lot of people watching, not a lot of people dead » (Philip Windsor, 1989, p. 275). Les cas, qui ne sont d'ailleurs pas rares, où aucune organisation ne revendique l'attaque par la suite ne sont pas en contradiction avec cela ( $c f$. Hoffman, 1997, et la discussion suivante, p. 7-17, par Pluschinsky et Rapoport).

Sans média de masse (aujourd'hui : sans télévision), point d'efficacité pour le terrorisme! Mais il peut exister un terrorisme efficace sans qu'il y ait de blessés ou de morts. C'est bien là la différence marquée entre le terrorisme et le bombardement de cibles non militaires (Belgrade 1941, Hiroshima 1945, etc.). Dans le cas de ces bombardements, les morts devaient convaincre la puissance adversaire de capituler, sinon toute sa population comme base humaine des organisations militaires risquait d'être anéantie. Dans le cas d'un attentat attribué à une organisation basque, palestinienne ou à al-Qaïda, la communication est comprise d'une manière bien différente: «Nous sommes toujours là ! » À la puissance ennemie: "Trouvez comment éviter de telles attaques. » À tous les sympathisants : "Joignez-vous à nous ou aideznous! » Évidemment, faire sauter un building sans faire de blessés ni de morts peut être un moyen bien plus adapté à cette sorte de protestation que de risquer la vie de dizaines ou de centaines de personnes. Loin de «choisir» des victimes humaines, la stratégie terroriste choisit d'impressionner la population (p. ex., LeVine, 1997). Cet objectif peut être atteint parfois sans faire de victimes. La quantité de corps humains dépend aussi de ce plan; elle n'est pas calculée comme dans le cas des bombardements de large périmètre où "plus de morts $=$ plus de chances de capitulation précoce ».

\section{LA PEUR, LA MENACE ET L'INNOCENCE DES VICTIMES}

La peur de la mort, généralisée dans la communication politique, peut être inspirée par toute violence organisée de type guerrier, génocidaire ou terroriste, les seules exceptions étant la violence d'un pouvoir judiciaire et policier, modérée par le droit libéral, ou bien la violence d'une organisation maffieuse, restreinte par la confiance et la concurrence. Autrefois, on avait l'impression que le mouvement pour la paix considérait qu'un monde sans violence militarisée était forcément un monde sans peur collective. Mais lier cette notion de paix à la notion de guerre (ou même de guerre entre des États nationaux) n'est plus convaincant pour beaucoup de monde. D'autres mouvements sociaux sont apparus en défense des droits de la personne contre des régimes génocidaires et même en simple défense des victimes du terrorisme. La communication ne peut plus s'empêcher de prendre en compte la particularité des violences guerrières, génocidaires et terroristes. "Une guerre contre le génocide», " une guerre contre le terrorisme », voilà des slogans qui laissent le mouvement pour la paix sans défense générale. Il ne lui reste que des arguments ad hoc: "C'est pas contre le génocide (ou le terrorisme), c'est parce qu'il y a du pétrole. » Les dangers du génocide, les dangers du terrorisme engendrent la peur et sont traduits en risque de non-intervention. La guerre, qui elle-même était si souvent la source de la peur, est alors considérée comme le moindre des maux.

Il y a un sens étroit du mot menace qui fait qu'on peut en réserver l'usage à désigner un état de choses dans lequel ego (soit un individu, soit une organisation) dit: « Si je ne fais pas ceci, quelque chose à connotation négative va se passer. » Hannah Arendt (1953) a même donné ce sens au mot peur, peur qui selon Montesquieu règne sous le régime de la tyrannie. Selon Arendt, il n'y a pas de valeur pratique de la peur dans un régime totalitaire parce que ceux qui seront victimisés ne peuvent l'éviter par des actes quelconques, étant victimes de lois dites naturelles (nazisme) ou sociales (stalinisme). On peut dire au moins que les actions génocidales sont exemptes de menaces (dans un sens étroit) parce que les victimes sont tuées pour ce qu'elles «sont»: tutsis, musulmans, etc., et non pas parce qu'elles résistent (et sont menacées pour cela : «Allez-vous en, sinon on vous tue !»). Les menaces du terrorisme ne sont pas dirigées vers ceux qui se trouvent victimes, mais contre des organisations - comme dans le cas d'une guerre "normale», non terroriste. Pour la communication du danger et de l'anxiété, cela n'a pas d'importance. La peur est peut-être plus grande quand ceux qui ne peuvent rien faire pour y échapper sont la cible. Dans une guerre « normale», l'armée (l'armée de guérilla incluse) ou son gouvernement n'engendrent pas l'anxiété. C'est l'héroïsme qui est demandé et cette approche du danger n'est pas compatible avec la peur.

En ce qui a trait à l'innocence des victimes, cette sémantique qui doit servir à marquer une différence entre guerre et génocide n'a jamais empêché de hiérarchiser les victimes, c'est-à-dire d' « héroïser » ceux qui sont tués dans le contexte d'une guerre. Michel Chaumont a montré dans une étude admirable (1997) qu'après le retour des détenus d'Auschwitz, ce sont les résistants qui ont occupé l'espace public dans les pays francophones d'Europe tandis que les rescapés qui avaient échappé à la mort qui leur était destinée parce qu' «ils étaient ce qu'ils étaient » ont été escamotés. Dans les années 1960, cette hiérarchie morale (et légale !) s'est renversée. Naturellement, considérer les morts d'une attaque terroriste comme des êtres qui sont en dehors de la distinction action / innocence ne peut pas servir à les mettre en dehors d'une communication hiérarchisante. Dans le cas de l'attaque contre le World Trade Center, ces morts ont été placés au-dessus des morts de la guerre contre le terrorisme en Afghanistan.

\section{OBSERVATION SOCIOLOGIQUE}

Une sociologie qui établit une distinction dans la communication entre sémantique et structure sociale, et qui distingue également entre plusieurs sphères de communication est encore moins utile à une démarche de moralisation convaincante. Elle montre comment la communication dépend du point d'observation. Quand la sémantique d'un discours juridique ou d'un discours de science politique ne parle de guerre qu'en cas de conflits entre États nationaux et utilise le terme de terrorisme (ou de low intensity conflict) pour désigner les tueries qui ne correspondent pas à cette image, la communication violente elle-même (qui fait partie de la structure sociale) peut se définir autrement : comme guerre, communication militaire. Quand la sémantique juridique dit «terrorisme», la sémantique du champ politique peut dire "guerre de libération » avec "les armes des pauvres». Quand la religion dit «Jihad», traduit par "guerre ", la politique, même celle qui a sa source dans une population musulmane (si 
cette politique est distincte de la religion, comme dans des structures modernes) peut dire « terrorisme».

Il n'existe pas de "vérité » sémantique. Et il n'existe pas d'utilité qui englobe plusieurs sphères de communication. La sociologie n'a pu observer jusqu'ici une telle utilité, et l'utilité sociologique n'est que celle d'un champ de la société moderne: celui de la science, qui elle-même n'est en rien un observatoire privilégié.

Est-ce que ce pluralisme d'observations et de perspectives d'observation est une réalité - ou est-ce qu'il est créé par la sociologie? Niklas Luhmann nous donne une réponse à cette question épistémologique ${ }^{3}$ : La réalité, dit-il, est créée dans ce qu'on peut nommer résistance de la communication contre la communication (Luhmann, 1996a). Toute communication est construite (communication sémantique ou non), mais il existe de la communication qui réussit à résister à des tentatives communicatives de dire: "non, ce phénomène est différent». Pour ma part, je pense que l'observation de la distinction, en tant que structure sociale, entre deux catégories de tueries organisées peut être vue comme générant une réalité (dans le sens que propose Luhmann) : les modes d'extermination qu'on peut qualifier de guerrier et génocidal. Mais la distinction sémantique, dans le mode de la guerre, entre la guerre dite normale et le terrorisme peut être contestée plus facilement ${ }^{4}$.

Avec ces trois distinctions, on peut essayer d'observer la communication d'anxiété. Ce n'est qu'une hypothèse plausible que d'avancer qu'en cas de guerre non terroriste, des normes, surtout la norme de l'héroïsme, empêchent la communication publique de peur. Mais cela n'exclut pas qu'on puisse observer la peur dans les familles et dans les unités de combat, dans la protestation d'un mouvement pour la paix (s'il n'est pas écrasé) et peut-être aussi en dehors de la communication - dans l'état psychique des êtres humaint ${ }^{5}$.

\section{Bibliographie}

ARENDT, Hannah (1953). "Ideologie und terror », dans Offener Horizont. Festschrift fuer Karl Jaspers, Munich, Piper, p. 229254.

ARONSON, I. Michael (1990). Troubled Waters. The Origins of the 1881 Anti-Jewish Pogroms in Russia, Pittsburgh, Univ. of Pittsburgh Press.

BOUCHEREAU, Philippe (1999). «Du génocide et de la guerre », L'intranquille, vol. 4-5, p. 137-161.

CHAUMONT, Jean-Michel (1997). La concurrence des victimes. Génocide, identité reconnaissance, Paris, Éd. La Découverte.
DAMMANN, Klaus (2003). "Women, children, older people - Genocide, warfare, and the functional differentiation of society", dans Grazyna Skapska et al. (dir.), The Moral Fabric in Contemporary Societies. Annals of the International Institute of Sociology, Leiden et Boston, Brill.

DAMMANN, Klaus (2001). Die Armenische Katastrophe - Genozid, Pogromwelle, Krieg, Bestrafung oder was sonst? Eine soziologische Untersuchung semantischer Opportunitaetsstrukturen zur Leugnung von Voelkermord, Bielefeld, Universitaet: Bielefelder Arbeiten zur Verwaltungssoziologie, 2001/1.

FEIN, Helen (1993). Genocide. A Sociological Perspective, $2^{\mathrm{e}}$ éd., Londres, Sage.

HABERMAS, Juergen et Niklas LUHMANN (1971). Theorie der Gesellschaft oder Sozialtechnologie - Was leistet die Systemforschung? Frankfurt am Main, Suhrkamp.

HOFFMAN, Bruce (1997). «Why terrorists don't claim credit », Terrorism and Political Violence, vol. 9, $\mathrm{n}^{\circ} 1$, p. 1-6.

LE VINE, Victor T. (1997). «On the victims of terrorism and their innocence", Terrorism and Political Violence, vol. 9, $\mathrm{n}^{\circ}$ 3, p. $55-62$.

LIFTON, Robert Jay et Eric MARKUSEN (1990). The Genocidal Mentality. Nazi Holocaust and Nuclear Threat, New York, Basics.

LUHMANN, Niklas (2002). "The cognitive program of constructivism and the reality that remains unknown", dans William Rasch (dir.), Theories of Distinction. Redescribing the Description of Modernity, Stanford, Stanford University Press, p. 128-152.

LUHMANN, Niklas (2000). "Answering the question: what is modernity? An interview with Niklas Luhmann ", dans William Rasch, Niklas Luhmann's Modernity. The Paradoxes of Differentiation, Stanford, Cal., Stanford University Press, p. 195-221.

LUHMANN, Niklas (1996a). «La science de la société: Questions à Niklas Luhmann. Entretien par André Kieserling et Georg Kruecken », Recherches Sociologiques, vol. 27, $\mathrm{n}^{\circ} 2$, p. 5-23.

LUHMANN, Niklas (1996b). "Complexity, structural contingencies and value conflicts », dans Paul Heelas, Scott Lash et Paul Morris (dir.), Detraditionalization. Critical Reflections on Authority and Identity, Cambridge, Mass. et Oxford, UK, Blackwell, p. $59-71$

LUHMANN, Niklas (1993). Risk: A Sociological Theory, Berlin et New York, De Gruyter.

MARTUCCELLI, Danilo (1999). Sociologies de la modernité. L'itinéraire du $X X^{e}$ siècle, Paris, Gallimard.

SCHMID, Alex P. et Albert J. JONGMAN (1988). Political Terrorism. A New Guide to Actors, Authors, Concepts, Data Bases, Theories and Literature. Édition revue, augmentée et mise à jour, Amsterdam et New Brunswick, North-Holland Publishing et Transaction.
SILKE, Andrew (1996). "Terrorism and the blind men's elephant», Terrorism and Political Violence, vol. 8, n ${ }^{\circ} 3$, p. 12-28.

STAEHELI, Urs (1997). "Exorcising the "popular" seriously: Luhmann's concept of semantics", International Review of Sociology, vol. 7, p. 127-145.

STICHWEH, Rudolf (2000). "Semantik und sozialstruktur: Zur logik einer systemtheoretischen unterscheidung», Soziale Systeme, vol. 6, p. 237-250.

WALDMANN, Peter (1989). Terrorismus. Provokation der Macht, Muenchen, Gerling Akademie.

WHEELER, Everett L. (1991). «Terrorism and military theory: An historical perspective", Terrorism and Political Violence, vol. 3, $\mathrm{n}^{\circ} 1$, p. 6-33

WINDSOR, Philip (1989). "Book review», Terrorism and Political Violence, vol. 1, $\mathrm{n}^{\circ} 2$, p. $274-275$.

\section{Notes}

1. C'est Jürgen Habermas, qui en effet a été construit comme l'antipode de Niklas Luhmann, depuis leur échange en 1968-1971 sur la "Théorie de la société ou technologie sociale?» (Habermas et Luhmann, 1971). Ironiquement, une «théorie de la société » est attribuée aujourd'hui à Luhmann (culminant dans son «Die Gesellschaft der Gesellschaft », Frankfurt am Main, 1997), Habermas est vu davantage comme philosophe et essayiste politique, et la sociologie de Bourdieu, qui renonce presque comme celle de Max Weber à une notion de "société », est de plus en plus considérée comme théorie en concurrence (voir p. ex. Martuccelli, 1999). Il $\mathrm{y}$ a maintenant trois livres et une vingtaine d'articles de Luhmann traduits en français.

2. À part les deux distinctions directrices (organisation comme auteur ou non et choix de victimes par critères ascriptifs ou d'action), il y a une dizaine de distinctions supplémentaires dans la sémantique guerre / génocide qui ne se trouvent pas seulement dans un discours négationniste (Dammann, 2001).

3. Pour une version élaborée de son approche épistémologique, $c f$. Luhmann, 2002.

4. La sémantique du terrorisme englobe, sauf les distinctions directrices mentionnées, une vingtaine de distinctions supplémentaires; pour une recherche sur les définitions, voir Schmid et Jongman, 1988. Par exemple, on peut utiliser la distinction entre guérilla en campagne et guérilla en ville et ne qualifier de «terrorisme» que les actes de guérilla urbaine.

5. Je remercie Juliette Bockslaff, Université de Bielefeld, et Diane Laflamme, Université du Québec à Montréal, pour avoir amélioré mon français d'étudiant. 